\title{
A suitable k-epsilon model for CFD simulation of pump-around jet mixing tank with moderate jet reynolds number
}

\author{
Satapan Phapatarinan ${ }^{1}$, Eakarach Bumrungthaichaichan ${ }^{1}$, and Santi Wattananusorn ${ }^{1, *}$ \\ ${ }^{1}$ Department of Chemical Engineering, Faculty of Engineering, \\ King Mongkut's Institute of Technology Ladkrabang, Bangkok, Thailand
}

\begin{abstract}
This paper presents the appropriate turbulence model for predicting the overall mixing time inside an open $45^{\circ}$ inclined side entry pump-around jet mixing tank with moderate jet Reynolds number of about 17,515 . The model was carefully developed by using appropriate hexahedral grid arrangement and proper numerical methods. The two different k-epsilon turbulence models, including realizable k-epsilon model and low Reynolds number k-epsilon model, were simulated. The overall mixing times predicted by these turbulence models were compared with the previous data reported by Patwardhan (Chem. Eng. Sci. 57 (2002) 1307-1318). The results revealed that the low Reynolds number k-epsilon model was a suitable model for predicting the overall mixing time of jet mixing tank with moderate jet Reynolds number.
\end{abstract}

\section{Introduction}

Jet mixing is an important mixing device, which was firstly proposed by Fossett and Prosser [1]. It provides the various advantages, including simple design with non-moving part, inexpensive operating cost, and easy installation and maintenance. The liquid recirculation and entrainment inside the tank are driven by high velocity jet to mix the different components.

The jet mixing tanks were experimentally studied to achieve the mixing time correlations. However, these correlations were case specific [2]. So, the computational fluid dynamics (CFD) has been adopted to address this problem and to illustrate the details of fluid flow and mixing inside the vessel.

Over two decades or so, there are many different CFD models for pump-around jet mixing tanks. For high jet Reynolds number $\left(\operatorname{Re}_{j}=\rho d_{j} U_{j} / \mu\right.$, where $\rho$ is fluid density, $d_{j}$ is jet nozzle diameter, $U_{j}$ is jet discharge velocity, and $\mu$ is fluid viscosity), most of these successful CFD models underpredicted the overall mixing times because of the overprediction in total momentum available for mixing due to the flat top liquid surface assumption as reported by Bumrungthaichaichan et al. [3]. However, Patwardhan [4] showed that the overall mixing time for moderate jet Reynolds number was overpredicted. Furthermore, Ghahremanian and Moshfegh [5] reported that the appropriate turbulence model should be selected for predicting the free turbulent round jet with low Reynolds number.

So, in this work, the two different k-epsilon turbulence models, including realizable $\mathrm{k}$-epsilon model (RKE) and low Reynolds number k-epsilon model (LRKE), were simulated to investigate the proper turbulence model for predicting the overall mixing time inside the pump-around jet mixing tank with moderate jet Reynolds number because there is no turbulence model which is valid for all flow situations. Further, the previous CFD works only studied the suitable turbulence model for high Reynolds number jet mixing tank.

\section{Description of CFD modelling}

\subsection{Jet mixing tank}

The studied system was an open $45^{\circ}$ inclined side entry pump-around jet mixing tank reported by Patwardhan [4]. The tank diameter and height of tap water were 0.5 $\mathrm{m}$. The jet nozzle diameter and tank outlet pipe diameter were $0.008 \mathrm{~m}$ and $0.0381 \mathrm{~m}$, respectively. The schematic of pump-around jet mixing tank is shown in Fig. 1.

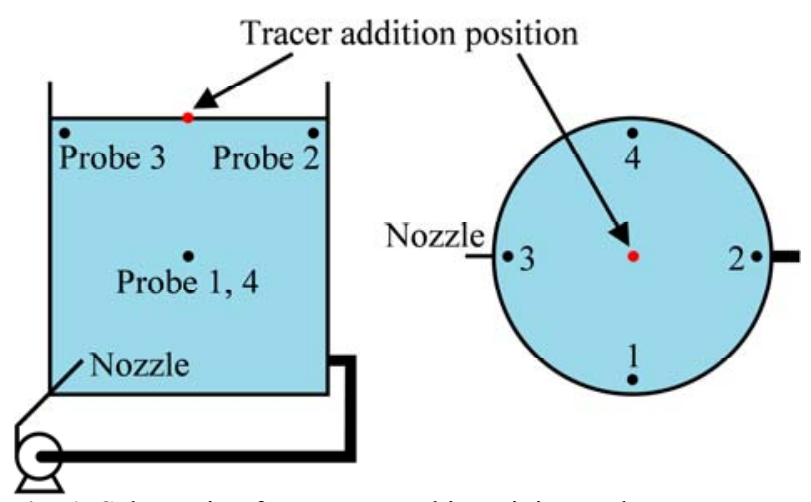

Fig. 1. Schematic of pump-around jet mixing tank

\subsection{Grid generation}

The three-dimensional solid model and grid generation of this jet mixing tank were carefully performed by using 
GAMBIT 2.4.6. The hexahedral grid topology was selected to obtain the accurate results. Further, the flow domain was manually split into the several blocks by using the specified faces to achieve the appropriate grid arrangement. That is, the grids were controlled to align with the flow direction to minimize the truncation error and numerical diffusion. The grid generation of this mixing tank is shown in Fig. 2.

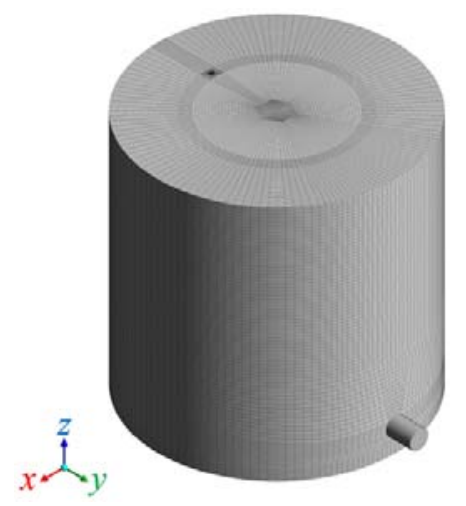

Fig. 2. Grid generation of pump-around jet mixing tank

\subsection{Governing equations}

For CFD simulation of jet mixing tank, the steady state jet flow and turbulence were achieved by using the Reynolds average equations for conservation of mass and momentum together with turbulence model. The steady state Reynolds average equation can be written in compact form as:

$$
\frac{\partial(\rho \boldsymbol{U} \phi)}{\partial x_{j}}=\frac{\partial}{\partial x_{j}}\left(\Gamma_{\varphi} \frac{\partial \phi}{\partial x_{j}}\right)+S_{\varphi}
$$

where $\boldsymbol{U}$ is mean velocity vector, $\phi$ is a universal dependent variable, $\Gamma_{\varphi}$ is the diffusivity, and $S_{\varphi}$ is the source term. Further, the details of variables for continuity and momentum equations are represented in Table 1.

Table 1. Variables for continuity and momentum equations

\begin{tabular}{|c|c|c|c|}
\hline Equation & $\boldsymbol{\phi}$ & $\boldsymbol{\Gamma}_{\varphi}$ & $\boldsymbol{S}_{\boldsymbol{\varphi}}$ \\
\hline Continuity & 1 & 0 & 0 \\
\hline Momentum & $U_{i}$ & $\mu$ & $-\frac{\partial P}{\partial x_{i}}+\frac{\partial}{\partial x_{j}}\left(\mu_{t} \frac{\partial U_{i}}{\partial x_{j}}\right)+S_{M, i}$ \\
\hline
\end{tabular}

For turbulence models, the details of RKE and LRKE turbulence models were clearly described and reported by ANSYS Fluent Theory Guide [6] and previous work of Xin and Lei [7], respectively. By default, the LRKE turbulence model is hidden by ANSYS FLUENT. However, this model can be enabled by respectively typing the two additional commands in TUI, including define/models/viscous/turbulence-expert/low-re-ke? and yes [8].
In transient simulation of mixing time, the unsteady state species transport equations without chemical reaction [6] were solved and can be written as:

$$
\frac{\partial\left(\rho Y_{i}\right)}{\partial t}+\frac{\partial\left(\rho \boldsymbol{U} Y_{i}\right)}{\partial x_{j}}=\frac{\partial}{\partial x_{j}}\left(\left(\rho D_{i, m}+\frac{\mu_{t}}{\mathrm{Sc}_{t}}\right) \frac{\partial Y_{i}}{\partial x_{j}}\right)+S_{i}
$$

where $Y_{i}$ is the local mass fraction of species $i, D_{i, m}$ is the mass diffusion coefficient for species $i$ in the mixture, $\mathrm{Sc}_{t}$ is turbulent Schmidt number, $\mu_{t}$ is the eddy viscosity, and $S_{i}$ is the source term for species transport equations.

Further, in this paper, the $95 \%$ mixing time was used to evaluate the mixing time. Generally, the $95 \%$ mixing time is the time required for concentration $(c)$ to reach within $95 \%$ of the fully mixed value $(\bar{c})$ and can be written as:

$$
t_{95 \%}=\text { time for }\left|\frac{c-\bar{c}}{\bar{c}}\right| \leq 0.05
$$

Finally, the overall mixing time of jet mixing tank was evaluated by using arithmetic average of mixing times obtained by four different probe locations as depicted in Fig. 1.

\subsection{Boundary conditions}

For steady state simulation, the velocity-inlet and pressure-outlet boundary condition types were adopted at inlet and outlet, respectively. Whereas, for transient simulation, the recirculation-inlet and recirculationoutlet were used [3]. At inlet, the uniform jet discharge velocity of $2.2 \mathrm{~m} \cdot \mathrm{s}^{-1}$ was specified. Moreover, for turbulence quantities, the turbulence kinetic energy of $0.0726 \mathrm{~m}^{2} \cdot \mathrm{s}^{-2}$ and its dissipation rate of $2.794518 \mathrm{~m}^{2} \cdot \mathrm{s}^{-3}$ were directly imposed. At the tank wall and base, the noslip boundary condition and standard wall functions were adopted. Further, the flat top water surface was assumed by using symmetry boundary condition, e.g. the normal velocity and normal gradient of all variables are zero.

\subsection{Numerical methods}

For this work, the double precision pressure-based solver of ANSYS FLUENT finite volume CFD code was used to simulate jet mixing tank. The pressure-velocity coupling scheme was SIMPLE. The spatial discretization scheme for all quantities and temporal discretization scheme were second order upwind and first order implicit, respectively.

\subsection{Solution strategy}

In order to identify the solution convergence for steady state simulation, the area weighted average of the velocity magnitude at plane $x=0$ was monitored until it was constant. Further, the scaled residual of $10^{-5}$ for tracer was used as a convergence criterion for transient 
simulation. For unsteady state simulation of implicit solver, the time step size of $0.0025 \mathrm{~s}$ was selected $[3,9]$.

Moreover, in order to achieve the grid independent solutions, the velocity magnitude gradient adaption with the refine threshold of $10 \%$ of the maximum value [10] was used. From CFD work of Bumrungthaichaichan et al. [3], they suggested that the grids of CFD models should be adapted three times to obtain the accurate results. Hence, in this work, the final grid resolutions of the present CFD models were also achieved by adapting the CFD grids three times to obtain the grid independent solutions and to minimize or eliminate any uncertainties.

\section{Results and discussion}

As mentioned earlier, the two different k-epsilon models, including RKE and LRKE, were simulated to investigate the appropriate k-epsilon turbulence model for CFD simulation of open $45^{\circ}$ inclined side entry pump-around jet mixing tank with moderate jet Reynolds number. The predicted overall mixing times were compared with the previous results of Patwardhan [4] as shown in Table 2. Furthermore, the profiles of normalized concentration, which is generally defined as a ratio of the tracer concentration to the fully mixed value, and jet axial velocity contours of these turbulence models are represented in Fig. 3 and Fig. 4, respectively.

Table 2. Comparison of overall mixing times

\begin{tabular}{|c|c|c|}
\hline Model & Mixing time [s] & \% Error $^{\text {a }}$ \\
\hline EXP [4] & 57.00 & - \\
\hline CFD [4] ${ }^{\mathrm{b}}$ & 65.00 & 14.04 \\
\hline RKE & 65.15 & 14.30 \\
\hline LRKE & 54.59 & 4.23 \\
\hline
\end{tabular}

The percentage error is a ratio of absolute difference between the predicted overall mixing time and experimental mixing time to the experimental value.

b The overall mixing time was predicted by standard kepsilon (SKE) turbulence model and power law discretization scheme.

From Table 2, the results show that the overall mixing times predicted by RKE and SKE reported by Patwardhan [4], are slightly different and are about 14\% higher than that observed experimentally. Whereas, the LRKE underpredicts the overall mixing time by about $4 \%$.

In Fig. 3, the normalized concentration profiles at probe 1 of RKE and LRKE models are different. The start of normalized concentration profile simulated by LRKE is faster than that predicted by RKE, which indicates that the predicted convective transport of LRKE is greater than that obtained by RKE. The RKE shows two small peaks of normalized concentration profile at time of about $35 \mathrm{~s}$ and $55 \mathrm{~s}$. Whereas, the
LRKE does not clearly represent the peak of normalized concentration profile. According to the absence in peak of normalized concentration profile, it can be implied that the dispersive transport of LRKE is higher than RKE. Furthermore, the normalized concentration profile of LRKE is faster to reach the $95 \%$ mixing time criterion as compared to RKE.

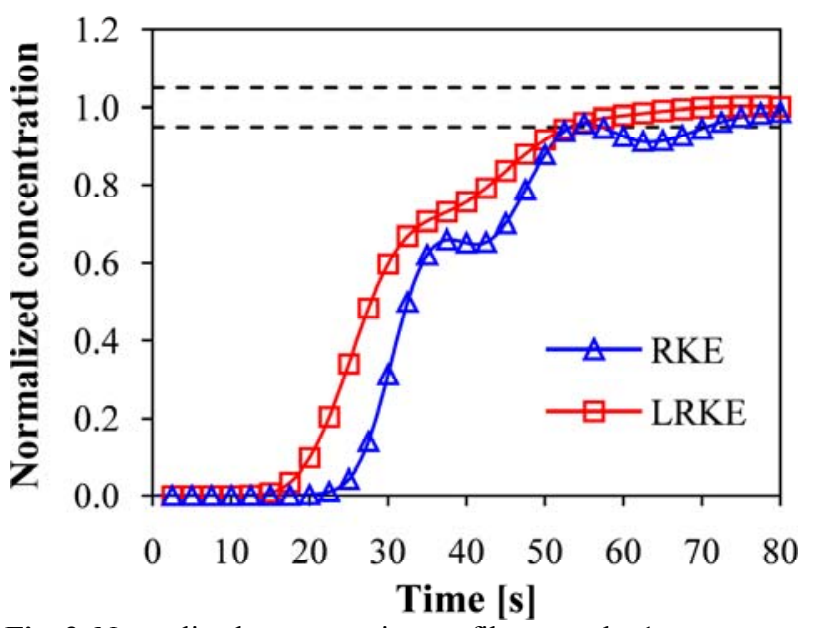

Fig. 3. Normalized concentration profiles at probe 1
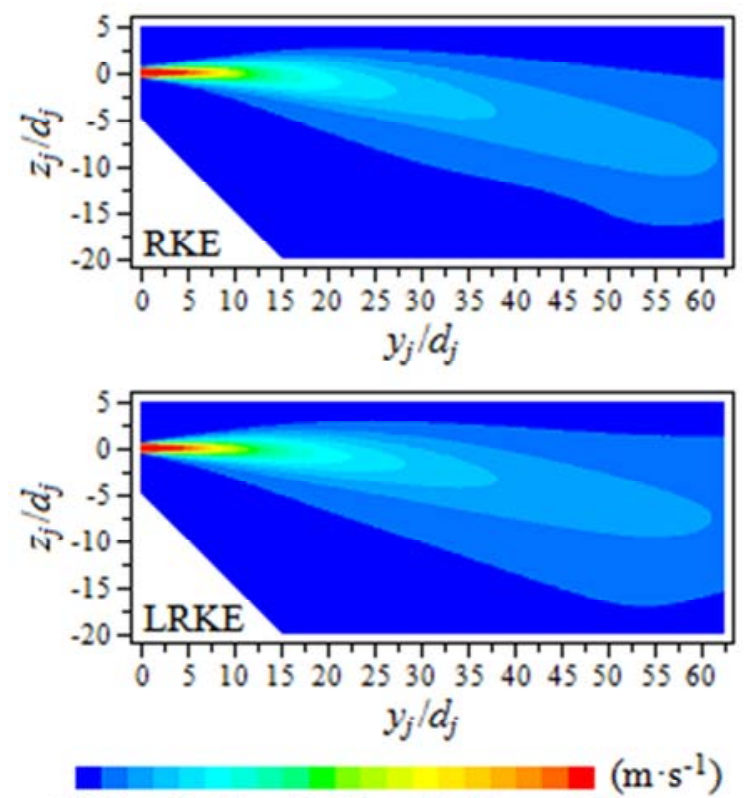

$\begin{array}{lllll}-0.08 & 0.49 & 1.06 & 1.63 & 2.20\end{array}$

Fig. 4. Contours of jet axial velocity at plane $x=0\left(y_{j}\right.$ and $z_{j}$ are jet longitudinal and jet radial distances.)

Fig. 4 represents the contours of jet axial velocity for two different $\mathrm{k}$-epsilon models. The results reveal that the decay of centreline velocity for RKE is faster than that observed by LRKE. Moreover, the jet spreading of LRKE is wider than RKE. Hence, the total momentum available for mixing of LRKE would be higher than RKE, which directly results in lower predicted overall mixing time. These results confirm that the convective and dispersive transports of LRKE are greater than those obtained by RKE.

Due to the lack of experimental data of concentration and velocity distributions inside the pump-around jet mixing tank, the overall mixing time was only compared 
with measured data [4] and used to select the appropriate turbulence model for jet mixing tank with moderate jet Reynolds number. Hence, the present predicted overall mixing time of jet discharge velocity of $2.2 \mathrm{~m} \cdot \mathrm{s}^{-1}$ and previous CFD data of Bumrungthaichaichan et al. [3] for other jet discharge velocities were plotted and compared with the experimental data of Patwardhan [4] as shown in Fig. 5.

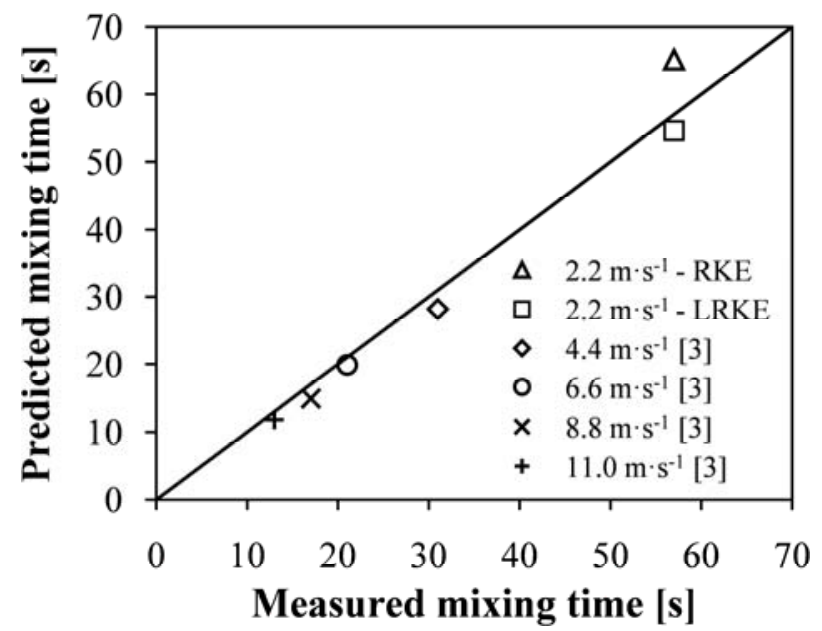

Fig. 5. Comparison of overall mixing times between simulations and experiments for different jet discharge velocities

From Fig. 5, the results show that the overall mixing times predicted by RKE are lower than experimental data for high jet Reynolds numbers $\left(U_{j} \geq 4.4 \mathrm{~m} \cdot \mathrm{s}^{-1}\right)$. For moderate jet Reynolds number $\left(U_{j}=2.2 \mathrm{~m} \cdot \mathrm{s}^{-1}\right)$, the RKE overpredicts the overall mixing time, however, LRKE underpredicts the overall mixing time.

From the previous work of Bumrungthaichaichan et al. [3], they reported that the underpredicted overall mixing time was due to the overprediction in total momentum available for mixing obtained by flat top liquid surface assumption. So, it can be summarized that the RKE can only be used for predicting the overall mixing time at high jet Reynolds number. Further, for moderate jet Reynolds number, the proper turbulence model is LRKE.

\section{Conclusion}

In this work, a CFD model was carefully developed to investigate the suitable $\mathrm{k}$-epsilon turbulence model for predicting the overall mixing time of pump-around jet mixing tank with moderate jet Reynolds number. The predicted overall mixing times of RKE and LRKE models were compared with the measured data of Patwardhan [4]. Due to the flat top liquid surface assumption, the proper k-epsilon turbulence model for predicting the overall mixing time inside this system was LRKE. For future work, in order to obtain the comprehensive CFD model for this system, the other low Reynolds number turbulence models should be employed and studied.
The authors would like to thank College of Advanced Manufacturing Innovation, King Mongkut's Institute of Technology Ladkrabang, Thailand for supporting the ANSYS FLUENT software.

\section{References}

1. H. Fossett, L.E. Prosser, Proc. I. Mech. E. 160, 224 (1949)

2. E. Bumrungthaichaichan, Korean J. Chem. Eng. 33(11), 3050 (2016)

3. E. Bumrungthaichaichan, A. Namkanisorn, S. Wattananusorn, J. Chin. Inst. Eng. (to be published)

4. A.W. Patwardhan, Chem. Eng. Sci. 57, 1307 (2002)

5. S. Ghahremanian, B. Moshfegh, J. Fluids Eng.Trans. ASME 136, 011201-1 (2014)

6. ANSYS Inc., ANSYS Fluent Theory Guide: Release 15.0 (ANSYS Inc., USA, 2013)

7. N. Xin, L. Lei, in 4th International Conference on Computer, Mechatronics, Control and Electronic Engineering, Hangzhou, China (2015)

8. ANSYS Inc., ANSYS Fluent Text Command List: Release 15.0 (ANSYS Inc., USA, 2013)

9. E. Bumrungthaichaichan, N. Jaiklom, A. Namkanisorn, S. Wattananusorn, Sci. Res. Essays 11(4), 42 (2016)

10. ANSYS Inc., ANSYS Fluent Tutorial Guide: Release 15.0 (ANSYS Inc., USA, 2013) 